\title{
Factors for Individualization of Therapeutic Exercises for the Design of Health-Enabling Technologies
}

\author{
Lena ELGERT ${ }^{\mathrm{a}, 1}$, Bianca STEINER ${ }^{\mathrm{b}}$, Birgit SAALFELD ${ }^{\mathrm{a}}$, \\ Michael MARSCHOLLEK ${ }^{\mathrm{a}}$ and Klaus-Hendrik WOLF ${ }^{\mathrm{a}}$ \\ a Peter L. Reichertz Institute for Medical Informatics of TU Braunschweig and \\ Hannover Medical School, Hannover, Germany \\ ${ }^{\mathrm{b}}$ Peter L. Reichertz Institute for Medical Informatics of TU Braunschweig and \\ Hannover Medical School, Braunschweig, Germany
}

\begin{abstract}
Designing health-enabling technologies (HETs) to support individualized physiotherapeutic exercises requires comprehensive knowledge of bio-psychosocial factors to be considered. Therefore, this review identified factors for individualization of therapeutic exercises in patients with musculoskeletal shoulder disorders in peer-reviewed articles searched in MEDLINE. The final full-text analysis included 16 of 335 search results and extracted nineteen main categories of individualization factors. The most frequently identified main categories include progression of exercises, exercise framework, and assessment. An iterative approach with constant reassessments represents the key principle for the process of individualization. Categories that are difficult to standardize were rarely mentioned, but should also be considered. The identified factors can improve the design-process of HETs by sensitizing developers, enable further formal modelling, and support communication between developers, physiotherapists, and patients.
\end{abstract}

Keywords. health-enabling technology, physical therapy, individualization, exercise

\section{Introduction}

Health-enabling technologies (HETs) are sensor-based information and communication technologies that promote a person's individual health, healthcare, and quality of life [1]. In physical therapy HETs can assist patients in performing exercises at home and support physiotherapists in assessments [2]. First studies indicate superiority of individualized compared to standardized non-individualized exercises in patients with musculoskeletal shoulder disorders [3]. HETs and treatment processes with HETs that assist patients and therapists with exercises should therefore promote individualized exercises and enable or support adaptation to the individual patient. Beyond this background, this literature review aimed to identify factors for individualization of therapeutic exercises in patients with musculoskeletal shoulder disorders to provide knowledge for designing HETs.

\footnotetext{
${ }^{1}$ Corresponding Author, Lena Elgert, Peter L. Reichertz Institute for Medical Informatics of TU Braunschweig and Hannover Medical School, Carl-Neuberg-Straße 1, 30625 Hannover, Germany; E-mail: lena.elgert@plri.de.
} 


\section{Methods}

The literature search and review process followed the PRISMA-ScR protocol [4]. Medical subject headings and text words related to the terms "upper extremity", "exercise and physical therapy", and "adaptation, progression, individualization, or personalization" constituted the search term in MEDLINE (via PubMed, on November 13, 2020). Two reviewers (LE and BSt) independently screened the 335 identified titles and abstracts against pre-defined inclusion and exclusion criteria. Reasons for exclusion were documented using the following main categories: 1 . No focus on musculoskeletal shoulder disorders $(n=184) ; 2$. No exercise $(n=24) ; 3$. Exercises not individualized $(n=82)$; 4. Other topics (e.g., prevalence of injuries during training) $(n=13)$; 5. Language other than English or German $(n=2)$. Full-text screening of the remaining 30 articles revealed five full-texts not reporting on individualized exercises. Nine full-texts were companion articles to the included main full-texts. The remaining 16 full-texts were incorporated in a systematic narrative synthesis based on a coding frame. The coding frame was built using a mix of concept- and data-driven-approaches [5] with some predefined main categories using the qualitative data analysis software MAXQDA (version 20.1.1; VERBI GmbH). The identified categories were illustrated in a code tree.

\section{Results}

Sixteen articles describing 15 projects were included [6-21]. One project was best represented by two complementing publications [19] and [20]. The 16 articles were published between 2007 and 2020. Treatment duration ranged from a single treatment to 5 months. Twelve weeks was the most common treatment period $(n=6)$. Target group were patients with musculoskeletal shoulder disorders (e.g., subacromial impingement syndrome). The number of patients assessed ranged from one to 708 with a median of 90. Different study types were present ranging from the evaluation of a HET to case reports and qualitative interview studies to randomized controlled trials.

Different types of individualization were identified. Besides selecting, personalizing, and progressing exercises, creating exercises was mentioned as a type of individualization. However, a detailed description of the procedure for creating exercises was missing. Kromer et al. [17] reported to proceed in the sense of clinical reasoning and thus create exercises. Anton et al. [6] claimed that the interface of their HET supports the creation of new exercises, but did not elaborate or refer to expert knowledge.

The analysis yielded 19 main categories and 87 sub-categories for individualization of exercises. With 23 sub-categories occurring in 59 coded segments in 14 articles the progression of exercises was the most frequent main category. Progression of exercises was realized, for example, by different performance levels [10] with validated scales for perceived exertion or reported pain $[14,15]$. The second and the third most frequent main categories were assessment (34 coded segments, 10 articles) and exercise framework (22 coded segments, eight articles). Validated assessment tools, like the Western Ontario Shoulder Index [7], range of motion (ROM) [16], and compliance measures [7], were applied. Measurements, such as ROM, served both as decision criteria to decide whether a certain exercise can be started [7] and to evaluate whether an exercise is effective [6]. Assessments were performed with re-tests and thus served to adapt exercises to the patient both at the start of treatment and during the course of treatment [17,20,21]. The constant reassessment was inherent in the individualization process. 
Factors maintaining or contributing to a patient's problem could be leisure and sport activities [20] or the work place setting [17]. In Anton et al. [6], the exercise individualization was based, among other things, on formalized knowledge in an ontology on biomechanical aspects. Figure 1 shows the main categories with frequency.

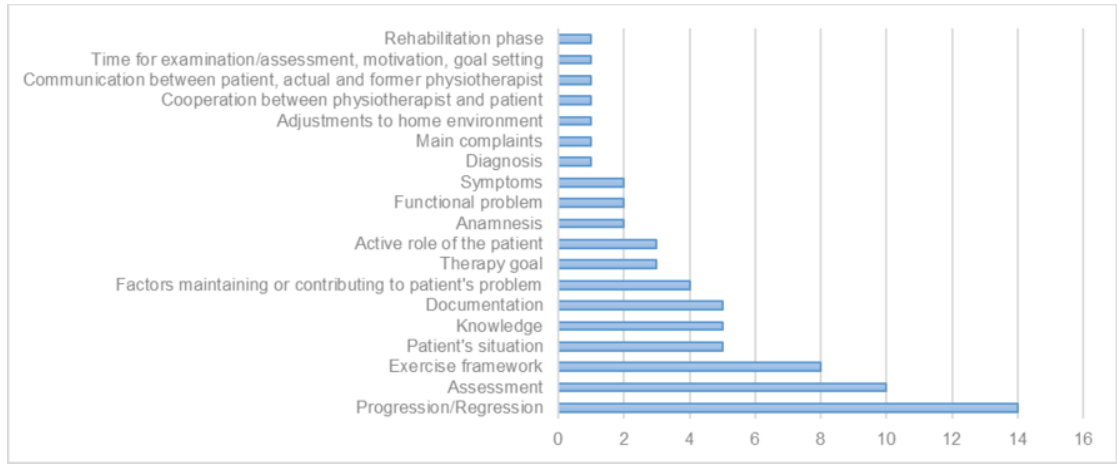

Figure 1. Main categories with frequency.

\section{Discussion and Conclusions}

Different individualization approaches of exercises are evident. Constant reassessment was identified as a key principle. Consequently, regardless of the selected approach, HETs should support or at least allow for reassessments. Since most rare individualization factors seem to be difficult to standardize, they may be underrepresented in this review of mostly highly standardized studies. However, that does not make them less important. Physiotherapy basically aims at sensomotoric self-determination of the individual and thus requires a meaningful frame of reference [22]. This means that exercises and therapy goals must be meaningful to the patient. Therefore, patients' everyday life, their environment, and their goals play a major role in the individualization of exercises. The rare as well as the frequent factors should be considered when designing HETs and physiotherapeutic treatment processes with HETs. Already in this limited literature search, the identified factors can provide insights, serve as a basis for further formal modeling and support the design-process of HETs. The results can sensitize developers and support communication between developers, physiotherapists, and patients. Further data collection and modelling, for example, in the form of an ontology, are planned to sufficiently support the design of HETs.

\section{References}

[1] Haux R, Koch S, Lovell NH, Marschollek M, Nakashima N, Wolf KH. Health-Enabling and Ambient Assistive Technologies: Past, Present, Future. Yearb Med Inform. 2016 Jun 30;Suppl 1(Suppl 1):S76-91.

[2] Elgert L, Steiner B, Saalfeld B, Marschollek M, Wolf KH. Health-Enabling Technologies to Assist Patients With Musculoskeletal Shoulder Disorders When Exercising at Home: Scoping Review. JMIR Rehabil Assist Technol. 2021 Feb 4;8(1):e21107.

[3] Roddy E, Ogollah RO, Oppong R, Zwierska I, Datta P, Hall A, Hay E, Jackson S, Jowett S, Lewis M, Shufflebotham J, Stevenson K, van der Windt DA, Young J, Foster NE. Optimising outcomes of exercise 
and corticosteroid injection in patients with subacromial pain (impingement) syndrome: a factorial randomised trial. Br J Sports Med. 2021 Mar;55(5):262-271.

[4] Tricco AC, Lillie E, Zarin W, O'Brien KK, Colquhoun H, Levac D, Moher D, Peters MDJ, Horsley T, Weeks L, Hempel S, Akl EA, Chang C, McGowan J, Stewart L, Hartling L, Aldcroft A, Wilson MG, Garritty C, Lewin S, Godfrey CM, Macdonald MT, Langlois EV, Soares-Weiser K, Moriarty J, Clifford T, Tunçalp Ö, Straus SE. PRISMA Extension for Scoping Reviews (PRISMA-ScR): Checklist and Explanation. Ann Intern Med. 2018 Oct 2;169(7):467-473.

[5] Schreier M. Qualitative content analysis in practice. London: SAGE; 2013. 272 p.

[6] Anton D, Berges I, Bermúdez J, Goñi A, Illarramendi A. A Telerehabilitation System for the Selection, Evaluation and Remote Management of Therapies. Sensors (Basel). 2018 May 8;18(5):1459.

[7] Bateman M, Smith BE, Osborne SE, Wilkes SR. Physiotherapy treatment for atraumatic recurrent shoulder instability: early results of a specific exercise protocol using pathology-specific outcome measures. Shoulder Elbow. 2015 Oct;7(4):282-8.

[8] Beaudreuil J, Lasbleiz S, Richette P, Seguin G, Rastel C, Aout M, Vicaut E, Cohen-Solal M, Lioté F, de Vernejoul MC, Bardin T, Orcel P. Assessment of dynamic humeral centering in shoulder pain with impingement syndrome: a randomised clinical trial. Ann Rheum Dis. 2011 Sep;70(9):1613-8.

[9] Bennell K, Coburn S, Wee E, Green S, Harris A, Forbes A, Buchbinder R. Efficacy and cost-effectiveness of a physiotherapy program for chronic rotator cuff pathology: a protocol for a randomised, double-blind, placebo-controlled trial. BMC Musculoskelet Disord. 2007 Aug 31;8:86.

[10] Christiansen DH, Frost P, Falla D, Haahr JP, Frich LH, Andrea LC, Svendsen SW. Effectiveness of Standardized Physical Therapy Exercises for Patients With Difficulty Returning to Usual Activities After Decompression Surgery for Subacromial Impingement Syndrome: Randomized Controlled Trial. Phys Ther. 2016 Jun;96(6):787-96.

[11] Crowell MS, Tragord BS. Orthopaedic manual physical therapy for shoulder pain and impaired movement in a patient with glenohumeral joint osteoarthritis: a case report. J Orthop Sports Phys Ther. 2015 Jun;45(6):453-61, A1-3.

[12] Eriksson L, Lindström B, Ekenberg L. Patients' experiences of telerehabilitation at home after shoulder joint replacement. J Telemed Telecare. 2011;17(1):25-30.

[13] Gleyze P, Georges T, Flurin PH, Laprelle E, Katz D, Clavert P, Charousset C, Lévigne C; French Arthroscopy Society. Comparison and critical evaluation of rehabilitation and home-based exercises for treating shoulder stiffness: prospective, multicenter study with 148 cases. Orthop Traumatol Surg Res. 2011 Dec;97(8 Suppl):S182-94.

[14] Ingwersen KG, Christensen R, Sørensen L, Jørgensen HR, Jensen SL, Rasmussen S, Søgaard K, JuulKristensen B. Progressive high-load strength training compared with general low-load exercises in patients with rotator cuff tendinopathy: study protocol for a randomised controlled trial. Trials. 2015 Jan 27; $16: 27$.

[15] Keene DJ, Soutakbar H, Hopewell S, Heine P, Jaggi A, Littlewood C, Hansen Z, Barker K, Hamilton W, Carr AJ, Lamb SE. Development and implementation of the physiotherapy-led exercise interventions for the treatment of rotator cuff disorders for the 'Getting it Right: Addressing Shoulder Pain' (GRASP) trial. Physiotherapy. 2020 Jun;107:252-266.

[16] Kinsella R, Cowan SM, Watson L, Pizzari T. A comparison of isometric, isotonic concentric and isotonic eccentric exercises in the physiotherapy management of subacromial pain syndrome/rotator cuff tendinopathy: study protocol for a pilot randomised controlled trial. Pilot Feasibility Stud. 2017 Nov 14;3:45.

[17] Kromer TO, de Bie RA, Bastiaenen CH. Effectiveness of individualized physiotherapy on pain and functioning compared to a standard exercise protocol in patients presenting with clinical signs of subacromial impingement syndrome. A randomized controlled trial. BMC Musculoskelet Disord. 2010 Jun 9;11:114.

[18] Littlewood C, Ashton J, Mawson S, May S, Walters S. A mixed methods study to evaluate the clinical and cost-effectiveness of a self-managed exercise programme versus usual physiotherapy for chronic rotator cuff disorders: protocol for the SELF study. BMC Musculoskelet Disord. 2012 Apr 30;13:62.

[19] Roddy E, Zwierska I, Hay EM, Jowett S, Lewis M, Stevenson K, van der Windt D, Foster NE; SUPPORT trial team. Subacromial impingement syndrome and pain: protocol for a randomised controlled trial of exercise and corticosteroid injection (the SUPPORT trial). BMC Musculoskelet Disord. 2014 Mar $14 ; 15: 81$.

[20] Stevenson K, Jackson S, Shufflebotham J, Roddy E, Foster NE. Development and delivery of a physiotherapist-led exercise intervention in a randomised controlled trial for subacromial impingement syndrome (the SUPPORT trial). Physiotherapy. 2017 Dec;103(4):379-386.

[21] Warby SA, Ford JJ, Hahne AJ, Watson L, Balster S, Lenssen R, Pizzari T. Effect of exercise-based management on multidirectional instability of the glenohumeral joint: a pilot randomised controlled trial protocol. BMJ Open. 2016 Sep 12;6(9):e013083.

[22] Probst A. Modell der menschlichen Bewegung. In: Höppner H, Richter R, editors. Theorie und Modelle der Physiotherapie, Ein Handbuch. Bern: Hogrefe; 2018. p. 99-113. 